\title{
Elektrophoretische Isoenzym-Auftrennungen der Lactatdehydrogenase im Agargel
}

\author{
Von Chr. FröhLICH \\ Aus der Mediziniscben Universitätsklinik Tübingen (Direktor: Prof. Dr. H. E. Bock)
}

(Eingegangen am 13. Oktober 1964)

\begin{abstract}
Es wird eine Agargel-Elektrophorese zur Auftrennung der LDH-Isoenzyme beschrieben, die auf Arbeiten von WIEME zurückgeht. Bei Benutzung einer LKB-Immunelektrophorese-Einrichtung können bis zu 18 Untersuchungen gleichzeitig vorgenommen werden. Wegen der verhältnismäßig wenig kostspieligen und unkomplizierten apparativen Einrichtung und der guten qualitativen Auswertbarkeit der Elektrophoresebanden ist die Methode für klinische Untersuchungen geeignet. Eine Begrenzung der diagnostischen Aussagefähigkeit des Verfahrens ergibt sich aus der Gleichartigkeit der im Serum auftretenden LDH-Isoenzym-Muster bei Erkrankungen verschiedener Organe. Eine Zuordnung der im Hitzeinaktivierungsverfahren gewonnenen LDH-Fraktionen zu elektrophoretischen Enzymfraktionen ist nicht sicher möglich.
\end{abstract}

A method is described for the separation of $\mathrm{LDH}$-isoenzymes by agar gel electrophoresis, based on the work of WIEME. With a LKB-imunoelectrophoresis apparatus, at least eighteen determinations can be carried out simultaneously. The method is suitable for clinical investigation, because the apparatus is relatively cheap and simple and the electrophoresis bands give a good qualitative determination. Use of the method for diagnosis is limited since the serum-LDH enzyme pattern is the same for illnesses of different organs. Correlation of LDH-fractions from the heat inactivation method with those from the electrophoretic separation is uncertain.

Seitdem MeISTER 1950 (1) erstmals elektrophoretisch die Heterogenität der Lactatdehydrogenase (LDH) in Organextrakten feststellte und später insbesondere Vesell und BeARN (2) dieselbe Beobachtung an der Serum-LDH machten, ist dieses Phänomen von verschiedenen Untersuchern auch unter Anwendung sehr differenter Methoden reproduziert worden. - Inzwischen liegen eine ganze Reihe von Arbeiten vor, die sich mit der Auftrennung der Organ-LDH und den theoretischen Aspekten der Ferment-Heterogenität befassen $(3,4,5)$. Es ist jedoch anzunehmen, daß die Enzymfraktionierung als Hilfsmittel der klinischen Diagnostik noch nicht voll ausgeschöpft ist. Arbeiten von vorwiegend klinischem Charakter liegen bisher vor von RICHTERICH und Mitarbeitern (6), die die Serum-LDH von Patienten mit verschiedensten Erkrankungen elektrophoretisch im Stärkeblock auftrennten. Vom gleichen Arbeitskreis (7) wurden LDHIsoenzym-Auftrennungen auch in Trans- bzw. Exsudaten verschiedener Genese veröffentlicht.

Úber eine große Zahl, wenn auch klinisch noch weiter gestreuten Untersuchungsmaterials haben DUBAcH und VARIOKojỊs berichtet. Sie benutzten zur Bestimmung der LDH-Isoenzymspektren die Hitzeinaktivierungsmethode, die jedoch nur eine Auftrennung in 2 Isoenzymfraktionen erlaubt, eine hitzestabile und eine hitzelabile. Diese Fraktionen sind mit den sonst üblichen elektrophoretischen Isoenzym-Fraktionen nur begrenzt vergleichbar. Mit der gleichen Methode wurde von ihnen auch die Möglichkeit einer genaueren Differenzierung des Myocardinfarkts von anderen Erkrankungen, wie Angina pectoris, Lungenembolie u. a. durch LDH-Isoenzym-Bestimmungen untersucht (9). Auf ein umfangreiches klinisches Material stützen sich die vor kurzem veröffentlichten Untersuchungsbefunde von Woerner (10). Methodisch verwandte er eine Folien-
Hochspannungs-Elektrophorese. WOERNER betont aufgrund seiner Befunde die Bedeutung der LDH-Isoenzym-Bestimmung zur Klärung der Hyperbilirubinämie sowie - ähnlich den Ergebnissen Dubach's zur Spätdiagnose des Herzinfarktes. Interessante vergleichende Untersuchungen der Gewebs- und SerumLDH-Zusammensetzung sowie Studien über quantitative und qualitative Serum-LDH-Veränderungen bei Reifungsstörungen der erythrozytären Reihe und hämolytischen Erkrankungen wurden von Hess $(11,12)$ mitgeteilt. - Eine Anzahl weiterer Arbeiten beschäftigt sich hauptsächlich mit Details der Auftrennmethodik oder erörtert richtungweisende Einzelbefunde (13 bis 23).

Statistisch gesichertes Material, auf das sich eine klinische Diagnostik im Einzelfall stützen könnte, liegt nur in beschränktem Umfang vor. Wir kennen zwar die wesentlichen im Patienten-Serum auftretenden LDH-Isoenzymmuster, wissen jedoch noch ungenügend über die Gesetzmäßigkeiten ihres Auftretens und damit über die Dignität des Einzelbefundes. Dies dürfte seine hauptsächliche Ursache im apparativen und zeitlichen Aufwand der bislang beschriebenen Bestimmungsverfahren haben, wie z. B. Stärkeblock-, Stärke-Gel-, Hochspannungs-Elektrophorese, Säulenchromatographie, immunologische Methoden, Prüfung der Hemmbarkeit durch verschiedene DPN-Analoge, Messung der $\mathrm{pH}$ Optima und andere mehr. Eine Ausnahme macht die Hitze-Inaktivierungsmethode und m. E. das Absorptionsverfahren an Zellulose. Beide Methoden erlauben, wie oben erwähnt, nur eine Auftrennung in 2 Fraktionen. Für orientierende Untersuchungen dürften sie in den meisten Fällen ausreichend sein. Sicher werden jedoch bei ihrer Anwendung Spektren mit einer Erhöhung der mittelschnell wandernden Fraktionen, wie sie bei Bluterkrankungen $z u$ beobachten sind, nicht 
genügend scharf erfaßt. Auch weitere Differenzierungsmöglichkeiten, die sich aus einer 5-fach Fraktionierung ergeben, gehen bei ihnen verloren. Dieser Umstand dürfte noch insofern nachteilig ins Gewicht fallen, als die Bedeutung der einzelnen Isoenzymfraktionen für die Diagnostik vermutlich noch nicht voll bekannt ist. Um daher mit geringem technischen Aufwand ein möglichst großes Untersuchungsmaterial mit voller Fraktionierung zu erhalten, haben wir die vom Arbeitskreis WIEME in Isoenzym-Untersuchungen eingeführte Agar-Gel-Elektrophorese in der Weise variiert, daß damit in einem Arbeitsgang 18 Einzeluntersuchungen vorgenommen werden können. Als ElektrophoreseApparatur benutzen wir die zur Durchführung der Routine-Immunelektrophoresen übliche „LKB““-Einrichtung (Fa. Colora-Meßtechnik, Lorch/Württemberg).

\section{Methode}

Eine 1-proz. Agarlösung wird in Veronal-Natrium/ Barbitursäure-Puffer ( $\mathrm{pH}=8,8$; Ionenstärke $=0,06 \mu$ ) hergestellt. Der Agar wird in üblicher Weise verflüssigt. Mit der noch heißen Agarlösung werden Objektträger $(25 \times 75 \mathrm{~mm})$ mit $2 \mathrm{ml}$ bedeckt. Bei Benutzung der „LKB“-Immunelektrophoreserahmen kann dies für jeweils 3 Objektträger in einem Arbeitsgang erfolgen. Nach Erkalten wird in dem Agar-Gel zum Auftrag des Serums eine Vertiefung von $1,5 \mathrm{~cm}$ Länge quer zum Längsdurchmesser des Objektträgers und etwa $3 \mathrm{~cm}$ von seinem rechten Rand entfernt angelegt. Zur Elektrophorese wird der oben erwähnte VeronälNatrium-Barbitursäure-Puffer verwandt. Die elektrophoretische Laufzeit beträgt durchschnittlich 140 Min. bei 250 Volt und $28 \mathrm{mAmpère.} \mathrm{Der} \mathrm{Fortgang} \mathrm{der}$ elektrophoretischen Wanderung kann an einem Objektträger kontrolliert werden, auf dem mit Kongorot angereichertes Serum wandert. Die genügende Wanderungsweite wird an der zurückgelegten Strecke der so markierten Albumine abgelesen.

Nach Abschluß der Elektrophorese werden die Objektträger mit folgender Substratlösung bedeckt:

\begin{tabular}{lll}
\hline Natriumlactat & $(1 \mathrm{~m})$ & $15 \mathrm{~m} l$ \\
Phenacine methosulfat & $(2 \mathrm{mg} / \mathrm{m} l)$ & $15 \mathrm{~m} l$ \\
Natriumcyanid & $(0,1 \mathrm{~m})$ & $15 \mathrm{~m} l$ \\
Nitro-blau-tetrazolium & $(5 \mathrm{mg} / \mathrm{m} l)$ & $15 \mathrm{~m} l$ \\
Phosphat-Puffer $\mathrm{pH} 6.0$ & $(0,1 \mathrm{~m})$ & $25 \mathrm{~m} l$ \\
DPN & & $50 \mathrm{mg}$ \\
Aqua dest. & & $80 \mathrm{ml}$ \\
\hline
\end{tabular}

Zur Anfärbung der LDH-Fraktionen macht man sich deren enzymatische Fähigkeit in der Weise zunutze, $\mathrm{da} ß$ dem Enzym in der Färbelösung ein Überschuß Lactat angeboten wird, das zu Pyruvat oxydiert wird, wobei der Wasserstoff über eine Reihe von Zwischenträgern auf ein oxydiertes Tetrazoliumsalz übertragen wird. Dessen Reduktion fühtt zum Ausfall eines wasserunlöslichen, blaugefärbten Salzes.

Die Inkubation muß bei einer durchschnittlichen Dauer von 50 Min. in Dunkelheit und bei $37^{\circ}$ erfolgen. An- schließend werden die Agar-Gel-Streifen auf den Objektträgern im Wärmeofen bei $40^{\circ}$ getrocknet. Die endgültige Auswertung des Enzym-Elektropherogramms nehmen wir mit dem Zeiss-Extinktions-Schreiber II vor.

Im folgenden seien einige der mit dieser Methodik gewonnenen klinischen Untersuchungen aufgeführt. Zur Reproduzierbarkeitsprüfüng wurde ein Serum mit einer mäßigen Erhöhung der kathodisch wandernden Fraktionen IV und $\mathrm{V}$ benutzt, um die schwierige $\mathrm{Ab}$ trennung der LDH IV von der LDH V-Fraktion besser beobachten zu können ${ }^{1}$ ).

Tab. 1

Reproduzierbarkeitsprüfung der Methode nach $s=\sqrt{\frac{\overline{\Sigma\left(x_{1}-\bar{x}\right)^{2}}}{n}}$. $20 \mathrm{LDH}$-Auftrennungen des gleichen Serums im Agargel. Im Gegensatz zu den übrigen in der Arbeit geschilderten Untersuchungen war in den zu dieser methodischen Prüfung notwendigen Auftrennungen ein Elektrophoresepuffer der Ionenstärke 0,24 benutzt worden

\begin{tabular}{|c|c|c|c|c|c|}
\hline \multirow[b]{2}{*}{ Lfd. Nr. } & \multicolumn{5}{|c|}{$\begin{array}{l}\text { LDH-Isoenzymfraktionen } \\
\text { Werte in relativen Prozenten }\end{array}$} \\
\hline & I & II & III & IV & $\mathrm{V}$ \\
\hline 1 & 22 & 34 & 34 & 8 & 2 \\
\hline 2 & 17 & 32 & 39 & 10 & 2 \\
\hline 3 & 21 & 34 & 34 & 8 & 3 \\
\hline 4 & 22 & 33 & 35 & 8 & 2 \\
\hline 5 & 22 & 33 & 33 & 9 & 3 \\
\hline 6 & 22 & 34 & 33 & 8 & 3 \\
\hline 7 & 19 & 31 & 41 & 7 & 2 \\
\hline 8 & 16 & 26 & 43 & 11 & 4 \\
\hline 9 & 18 & 30 & 37 & 12 & 3 \\
\hline 10 & 21 & 34 & 39 & $\dot{5}$ & 1 \\
\hline 11 & 16 & 34 & 36 & 12 & 2 \\
\hline 12 & 16 & 35 & 41 & 6 & 2 \\
\hline 13 & 17 & 28 & 40 & 11 & 4 \\
\hline 14 & 16 & 34 & 41 & 8 & 1 \\
\hline 15 & 22 & 35 & 37 & 5 & 1 \\
\hline 16 & 20 & 33 & 37 & 8 & 2 \\
\hline-17 & 15 & 36 & 38 & 9 & 2 \\
\hline 18 & 16 & 34 & 38 & 10 & 2 \\
\hline 19 & 17 & 32 & 39 & 10 & 2 \\
\hline 20 & 18 & 30 & 36 & 10 & 6 \\
\hline $\bar{n}$ & 18,7 & 32,6 & 37,6 & 8,8 & 2,5 \\
\hline$s$ & 2,5 & 2,4 & 2,8 & 2 & 1,2 \\
\hline
\end{tabular}

Zum Beispiel des in Abbildung 1 abgebildeten Isoenzym-Musters im Normalserum muß erwähnt werden, daß die LDH-V-Fraktion nicht in jedem Serum wie hier nachweisbar ist. In dem von RichTERICH angewendeten Elutionsverfahren (7) wird die LDH IV und $\mathrm{LDH} \mathrm{V}$ in einer Komponente, der sogenannten "Gamma-LDH“ zusammengefaßt. In den Verfahren, die nach der Elektrophorese den DPNH-Abfall in einer aufgelegten Kontrollplatte mit einer Substratmischung messen $(16,17,18)$ und in Färbeverfahren, wie später auch WİME (19) und wir es benutzen,

1) Von den unter üblichen Bedingungen erhaltenen 21 Auftrennungen war 1 Elektropherogramm technisch mißlungen und wurde nicht berücksichtigt. 


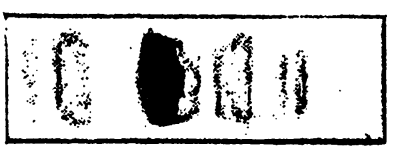

Normal-Serum

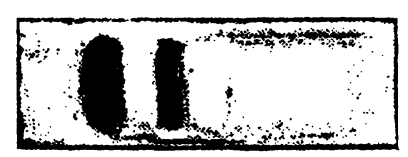

haemolytische Anaemie

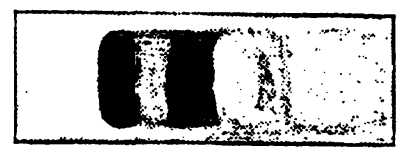

Myocardinfarkt

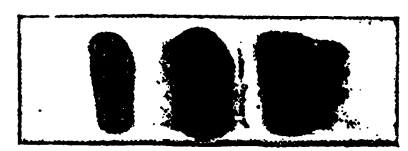

myeloische Leukaemic

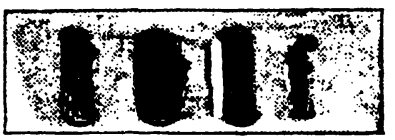

Colon-Carcinom Lebermetastasen?

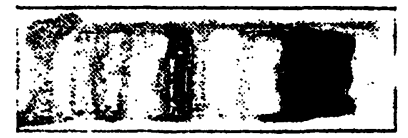

toxischer Leberschaden

Abb. 1

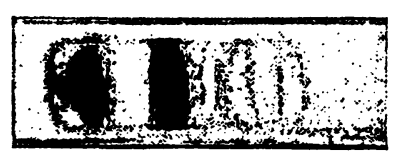

Normalserum

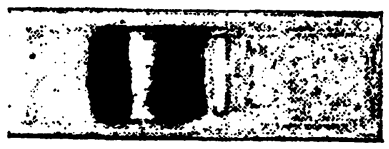

Myocardinfarkt

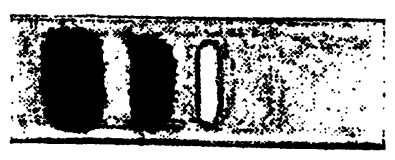

Haemolytische Anaemic

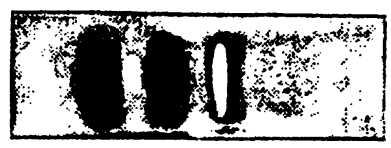

Akute toxische Anurie

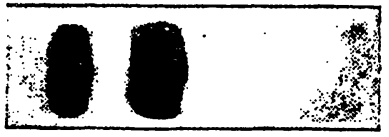

Neoplastische Durchwucherung des Herzmuskels

Abb. 2

enzymmuster bei klinisch weit divergierenden Diagnosen im Serum auftreten. - Derartige Spektren sind in Abbildung 2 aufgeführt.

Eine Differenzierung dieser Muster ergibt sich in den meisten Fällen eindeutig aus der klinischen Diagnostik. Wir konnten jedoch insbesondere bei Intoxikationen Erhöhungen der Gesamt-LDH-Aktivität beobachten, die durch Schädigung aller oben angeführten Organe hätten entstanden sein können. So auch in dem abgebildeten Fall einer akuten Anurie nach ChromIntoxikation. Aufgrund der eingehenden klinischen Diagnostik, die keinen Anhalt für eine hämolytische oder cardiale Komponente bot, mußten wir hier das Nierengewebe als im Vordergrund stehenden Ausgangspunkt der LDH-Erhöhung ansehen. Nach Abklingen der Anurie ergab sich bioptisch der Befund einer interstitiellen Nephritis.

Völlig unklar war anfänglich die Deutung des an fünfter Stelle aufgeführten Spektrums. Es handelte sich dabei um das Serum eines Patienten mit Bronchialkarzinom. Ein erythrozytärer oder nephrogener Ursprung der LDH-I-Erhöhung war aufgrund der vorliegenden klinischen Daten auszuschließen. Auch für eine Myocardbeteiligung lag kein Hinweis vor. Erst autoptisch ergab sich die Klärung des Befundes durch eine neoplastische Durchwucherung des Myocards. Einen gleich gelagerten Fall haben auch DuвAch und VARIokojrs (8) und jetzt auch Orelir und Dubach (9) beschrieben. Bei ihnen handelte es sich ebenfalls um eine neoplastische Durchsetzung des Myocards, ausgehend von Bronchialkarzinomen. 
Mit der gleichen oben beschriebenen Methodik haben wir auch Isoenzym-Untersuchungen in Erguß-Material vorgenommen. Eine Einengung des Ausgangsmaterials zur Untersuchung ist nicht erforderlich. - Von dem Arbeitskreis um RICHTERICH war in einer schon oben erwähnten Arbeit (7) die Möglichkeit mitgeteilt worden, durch Isoenzym-Analysen die benigne oder maligne Ätiologie eines Ergusses feststellen zu können. Ihr Untersuchungsmaterial bestand aus 30 Fällen. Die maligne Ätiologie eines Ergusses glaubten sie unter 3 Voraussetzungen annehmen zu können:

1. Die gamma-LDH liegt im Erguß in höherer Konzentration als im Serum vor.

2. Die gamma-LDH beträgt mehr als $80 \mathrm{IE}$.

3. Die gamma-LDH macht prozentual mehr als $30 \%$ der Gesamtaktivität aus.

Methodisch benutzten sie die Auftrennung im Stärkeblock mit anschließender Elution. Sie gewannen dabei $4 \mathrm{LDH}-F r a k t i o n e n$. Um vergleichbare Werte zu erhalten, muß bei Anwendung des von uns benutzten Verfahrens die Summe der LDH-IV- und LDH-VFraktion der von ihnen erwähnten "gamma-LDH“ gleichgesetzt werden. - Unter dieser Voraussetzung stimmen die in ihrer Arbeit veröffentlichten Befunde über Serum-LDH-Auftrennungen im wesentlichen mit den von WROBLEWSKI, WIEME und von uns gefundenen überein. Dies trifft jedoch nicht im gleichen $\mathrm{Maße}$ für die Exsudatauftrennung zu. Es wurden von uns 19 Pleura-Exsudate untersucht. Davon 11 benigner, 8 maligner Ätiologie. Natürlich ist die Zahl der Untersuchungen zu klein, um aus ihnen bindende Schlüsse ziehen zu können. Es konnte jedoch entgegen den RICHTERICH'schen Untersuchungsergebnissen beobachtet werden, daß auch in malignen Ergüssen ein LDHIsoenzym-Muster obne Betonung der IV. und V. Fraktion auftreten kann (s. Abb. 3, Beispiel 4 u. 5).

Demgegenüber war andererseits auch in benignen Exsudaten ein Muster mit Betonung dieser LDH-Fraktion nachzuweisen (s. Abb. 4, Beispiel 2 und 3). In Beispiel 3 und 4 war bei dem gleichen Patienten vermutlich infolge der Therapie eine Wandlung des Isoenzym-Musters zu beobachten. Es handelte sich um eine Pleuritis tuber- culosa, die zwischenzeitlich mit Corticoiden intrapleural behandelt worden war. Schon bei flüchtiger Betrachtung fällt die Verschiedenheit der einzelnen Isoenzym-Muster in der Gruppe der malignen Exsudate auf. Bei klinisch gleichem Prozeß (Bronchialkarzinom) findet sich einmal (Beispiel 1) eine starke Betonung der kathodisch wandernden Isoenzym-Fraktion (35\%), ohne daß eine Auftrennung in die IV. und V. LDH-Fraktion möglich wäre, ein andermal (Beispiel 2) ein Anstieg der LDHIV-Fraktion auf $73 \%$ bei fehlender LDH-III-Fraktion. Schließlich ist auch das Auftreten von nur 3 kaum den üblichen Mustern zuzuordnenden Fraktionen im Pleuraerguß eines Plasmozytomkranken bemerkenswert. $\mathrm{Da} \beta$ auch ein annähernd normales Verteilungsmuster wie in Beispiel 4 im Pleuraerguß bei Bronchialkarzinomen auftreten kann, wurde schon erwähnt.

Eine sichere Differenzierung der Erguß-Ätiologie war uns in Ubereinstimmung mit Dubach und Variokojrs (8) sowie WOERNER (10) anhand der RICHTERICH'schen Kriterien 2 und 3 nicht möglich. Leider liegen aus beiden erwähnten Arbeiten keine Angaben zu dem von RICHTERICH am stärksten bewerteten Kriterium 1 vor. In den von uns analysierten 10 Fällen mit benignen entzündlichen Ergüssen ließ sich jedoch zweimal auch dieses Malignitätszeichen als positiv nachweisen. - Es muß der Analyse eines größeren Untersuchungsmaterials vorbehalten bleiben, zu klären, inwieweit die histologische bzw. histochemische Differenzierung eines Tumors, seine Wachstumsausbreitung, insbesondere die Kommunikation mit dem Erguß sowie nicht zuletzt die Terapie Einfluß auf die Gestaltung des IsoenzymSpektrums nimmt.

Abschließend sei ein Vergleich der Methoden zwischen der LDH-Isoenzymbestimmung mit Hilfe der Hitzeinaktivierung - wie sie hauptsächlich von DuBACH angewendet wird - und der hier beschriebenen AgarGel-Elektrophorese angeführt. Wir haben dabei das LDH-reiche Serum eines Patienten mit einem in die Leber metastasierenden Melanom ausgewählt, um die hitzeinstabilen LDH-Fraktionen IV und V in genügend hoher Aktivität nachweisen zu können. Das Serum wurde entsprechend den Angaben DuBACH's (8) 30 Min. in Wasser verschiedener Temperatur inkubiert. - Der

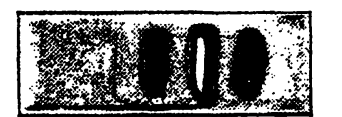

Pleura-Erguß bei:

Bronchial-Ca.

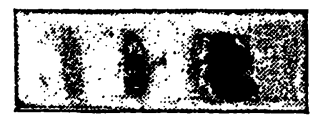

Bronchial-Ca.

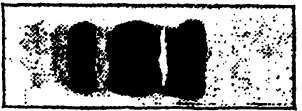

Plasmocytom

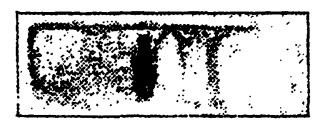

Bronchial-Ca.

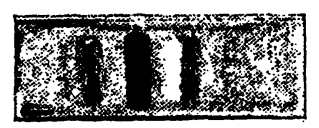

Pleura-Erguß bei:

Lungeninfarkt

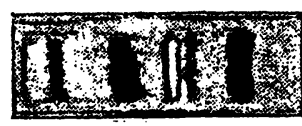

Pleuritis exsudativa

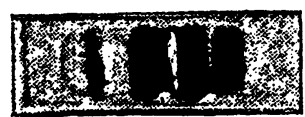

Pleuritis exsudativa 20.11 .

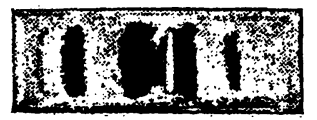

Pleuritis exsudativa 4.12. 
Tab. 2

\begin{tabular}{ccccccccccrrrr}
\hline & $\begin{array}{c}\text { Gesamt- } \\
\text { Aktivität } \\
\text { (IE) }\end{array}$ & $\begin{array}{c}\text { Aktivitäts- } \\
\text { abnahme } \\
(\%)\end{array}$ & I & II & III & IV & V & I & II & III & IV & V \\
\hline Ausgangsaktivität & 318 & & 10 & 17 & 33 & 15 & 30 & 5 & 54 & 105 & 48 & 95 & 16 \\
$40^{\circ}$ & 286 & 18 & 44 & 15 & 19 & 4 & 52 & 126 & 43 & 54 & 11 \\
$50^{\circ}$ & 188 & 41 & 24 & 47 & 15 & 10 & 4 & 45 & 88 & 28 & 19 & 8 \\
$57^{\circ}$ & 88 & 73 & 28 & 66 & 6 & - & - & 25 & 58 & 5 & - & - \\
$65^{\circ}$ & 56 & 82 & 44 & 56 & - & - & - & 25 & 31 & - & - \\
\hline
\end{tabular}

Gesamtaktivitätsverlust und seine Verteilung auf die einzelnen LDH-Fraktionen ist in Tabelle 2 dargestellt. Das Ergebnis beider Untersuchungen wäre das gleiche: Hitzelabilitätsprüfung: $\mathrm{SLDH}_{1}: \mathrm{SLDH}_{5}=18: 72=$ starkes Uberwiegen der hitzestabilen Fraktion (Aktivität des Testwertes bei Zimmertemperatur abzüglich des Testwertes bei $57^{\circ}$ ).

Elektrophoretisch: Betonung der kathodisch wandernden LDH-Fraktion IV, die 30\% der Gesamtaktivität ausmacht (=95 IE).

Bei Anwendung beider Untersuchungsmethoden müßte daher der Verdacht auf eine Hepatopathie ausgesprochen werden.

Während das Untersuchungsergebnis mit Hilfe der Hitzelabilitätsprüfung damit erschöpft wäre, ist elektrophoretisch das starke Hervortreten gerade der LDHIV-Fraktion auffallend. Bei Leberaffektionen fanden wir häufig beide langsam wandernden Fraktionen erhöht.
War nur eine dieser Fraktionen betroffen, dann ist es in der Regel die LDH-V-Fraktion. Der untersuchte Fall bietet somit bei Anwendung der elektrophoretischen Methodik ein durchaus auffallendes Isoenzym-Muster. Mit den Einschränkungen, die sich aus einer einmaligen Untersuchung zwangsläufig ergeben, müssen wir annehmen, daß eine Gleichsetzung hitzelabiler bzw. hitzestabiler Fraktionen mit Isoenzym-Fraktionen auf elektrophoretischer Basis nicht möglich ist. Es sind zwar die LDH-Fraktionen III, IV und V durch die Temperatureinwirkung besonders betroffen, gleichzeitig kommt es jedoch auch zu einer Aktivitätsabnahme in allen anderen Fraktionen. Insbesondere ist bei $57^{\circ}$ nicht nur eine Inaktivierung der IV. und V., sondern auch eines erheblichen Teils der III. Fraktion festzustellen. Daneben treten gleichzeitig Aktivitätsverluste in den beiden ersten Fraktionen auf, wobei jedoch deren Relation zueinander gewahrt bleibt.

\section{Literatur}

1. Meister, H., J. biol. Chemistry 184, 117 (1950). - 2. Veseli, E. S. und A. G. Bearn, Proc. Soc. exp. Biol. Med. 94, 96 (1957). - 3. Wieland, T., G. Pfleiderer, I. Haupt und W. Woerner, Biochem. Z., 332, 1 (1959?). - 4. WIELAND, T. und G. PfLEIDERER, Ann. N. Y. Acad. Sc. 94, 691 (1961). - 5. Wieland, T. und G. Pfleiderer, Advances Encymol. N. Y. 25, 329 (1963). 6. Zuppinger, K., R. Ruchterich und E. Rossi, Schweiz. med. Wschr. 92, 169 und 198 (1962). - 7. RICHTERICH, R., J. LOCHER, K. Zuppinger und E. Rossr, Schweiz. med. Wschr. 92, 918 (1962). - 8. Dubach, U. C. und D. Variakojrs, Schweiz. med. Wschr. 93, 1196 (1963). - 9. V. Orelli, A. und U. C. Dubach, Klin. Wschr. 42, 58 (1964). - 10. WöRNER, W., Med. Klin. 59, 11 (1964). - 11. Hess, B., Klin. Wschr. 38, 1080 (1960). - 12. Hess, B., Enzyme im Blutplasma, Georg Thieme Verlag, Stuttgart (1962). - 13. Hess, B., Verb. Dtsch. Ges. inn. Med., 70
(1964). - 14. Bär, U., E. Schmidt und F. W. Schmidt, Klin. Wschr. 41, 977 (1963). - 15. vaN DeR HeLM, H. J., Clin. Chim. Acta (Amsterdam) 7, 124 (1962). - 16. WIEME, R. J. und V. Maercke, Ann. N. Y. Acad. Sc. 94, 898 (1961). - 17. Wieme, R. J. und L. Demeulenaere, Acta gastro-enterol. Belg. 22, 69 (1959). - 18. Kamary, J. und Z. Zazvorka, Sci. Tools (Stockholm) 10, 2 (1963). - 19. Wreme, R. J., M. VAN SANDE, D. Karcher, A. Loewenthal und van der Helm, Clin. Chim. Acta (Amsterdam) 7, 750 (1962). - 20. Hill, B. R., Cancer Res., 21, 271 (1961). - 21. Blanchaer, M. C., Clin. Chim. Acta (Amsterdam) 6, 272 (1961). - 22. Markert, C. L. und H. URSPRUng, Development. Biol. 5,3 (1962). - 23. Dioguardi, N., A. Agostini und G. Froreliri, Encymol. biol. Clin., 2, 116 (1962/63).
Dr. Chr. Fröhlich Med. Universitätsklinik 74 Tübingen 